\title{
Reconstruction of gastrointestinal continuity by the gastric pull-up technique following en bloc pharyngo-laryngo-esophagectomy
}

\author{
Samy Saad, ${ }^{a}$ MD; Mohamed El-Shinawi, ${ }^{a}$ MD; Ahmed Alaa, ${ }^{a}$ FRCS(Ed); Ashraf \\ A. Mostafa, ${ }^{a}$ MD; Alaa El-Ashry, ${ }^{a}$ FRCS(I); Alaa Abbas Moustafa, ${ }^{a}$ MD FRCS(Eng) \\ FRCS (Ed); Mohamed Shehata, ${ }^{b}$ MD; Ahmed Adly, ${ }^{b} M D$ \\ a) Department of General Surgery, Ain-Shams University, Cairo, Egypt \\ b) Department of Otorhinolaryngology, Ain-Shams University, Cairo, Egypt
}

\begin{abstract}
Various methods of reconstruction have been applied after total pharyngo-laryngoesophagectomy in order to provide the continuity of the gastrointestinal tract. Gastric pull-up technique has recently become more popular because of the lower possibility of stricture and the need for a single anastomosis. This work is a combined effort among surgeons in the General Surgery \& Otorhinolaryngology departments in Ain-Shams University Hospitals studying the outcome of this technique after total pharyngo-laryngo-esophagectomy (PLE) in patients with resectable hypopharyngeal (postcricoid) carcinoma. We operated on twelve patients, in the period between August 2003 and April 2007. Oral intake was allowed on the 10th postoperative day following radiological confirmation of anastomotic integrity plus clinical well-being.

Four patients developed anastomotic leak (two major and two subclinical leaks detected radiologically) and postoperative neck hematoma occurred in one patient. Two complained of mild dysphagia due to anastomotic narrowing dilated endoscopically with Savary dilators.

All the surviving patients complained of regurgitation of various severities following the start of oral intake.

Total mortality was 5/12. Two patients succumbed to respiratory failure within 2 weeks of re-do for major leak. One patient re-explored for neck hematoma died following the sudden development of pneumothorax and consequent myocardial infarction and two died from severe electrolyte disturbance and multiorgan failure on 5th and 7th postoperative day, respectively.

Although this reconstruction is not without mortalities and morbidities, we consider it at worse a salvage procedure for already helpless patients.
\end{abstract}

\section{Introduction:}

Various methods of reconstruction have been applied after radical resections of pharynx, larynx and esophagus in patients suffering from postcricoid carcinomas in order to restore the continuity of the gastrointestinal tract. These methods are expected to provide the oral intake for appropriate nutrition while not influencing the standard of life negatively, i.e. as low as possible mortality and morbidity. Cervical skin grafts, the deltopectoral or myocutaneous grafts, gastric pull-up, colonic flaps, or jejunal free flaps are amongst the identified options. Cervical skin grafts are not widely applied not only because they include multiple steps, but also due to unacceptably common complications such as stricture and fistulization. ${ }^{1}$ Deltopectoral skin flap application with two steps ${ }^{2}$ has resulted in a high rate of complications and flap necrosis. ${ }^{3}$ Similar complications have occurred with the pectoralis major flap. ${ }^{4-6}$ Gastric pull-up method has recently become more popular because of the low possibility of stricture and the need for a single anastomosis. In parallel with the development of microsurgery techniques in recent years, jejunal free flap reconstruction is proposed as an alternative method against gastric pull-up, although the surgical technique is more complicated. ${ }^{7-9}$ Of these various techniques, gastric pull-up retains its place as the first option for reconstruction, at least for 
its relative simplicity and was our reconstructive method of choice in the present work.

\section{Patients and methods:}

We implemented this combined study in the period between August 2003 and April 2007, among the General Surgery and Otorhinolaryngology departments in AinShams University Hospitals. After taking an informed consent, twelve patients with proven postcricoid carcinomas underwent pharyngolaryngo-esophagectomy (PLE) followed by gastric pull-up reconstruction. Two patients received preoperative down staging radiotherapy for neck lymph nodes. All patients had the routine pre-operative assessment and laboratory work up, in addition to staging CT scan \& MRI. The otolaryngologist assessed the hypopharynx endoscopically under general anesthesia and collected biopsies for histopathological examination. Endoscopy also ruled out the existence of a second or concurrent malignancy and ensured that no skip lesions exist. Simultaneously in the same session, they determined whether the tumor invades the prevertebral fascia or not by examining its mobility over the latter. Thus, the tumor should be mobile (back and forth) when slid against the cervical vertebrae.

Before embarking on pharyngo-laryngoesophagectomy, all patients were given prophylactic antibiotic (ceftriaxone + metronidazole) one dose at the time of induction of anesthesia and twice daily for 5 days postoperatively. Low molecular weight heparin was started peri-operatively and continued for at least 7 days, postoperatively. The patients were nourished by TPN for 7-10 days preoperatively, and continued by feeding jejunostomy postoperatively. Contrast radiography was obtained on the 9 th postoperative day. Oral intake started on the 10th postoperative day following radiological confirmation of anastomotic integrity plus clinical well-being.

\section{Surgical technique:}

The otorhinolaryngology team performed pharyngo-laryngectomy and radical or modified neck dissections for resectable tumors Figure(1).

Intraoperative frozen section examination of the resection margins was not available. As the otorhinolaryngology team confirmed resectability, the general surgery team began laparotomy with upper abdominal midline incision. Following an extensive Kocher maneuver, the right gastric artery and the right gastroepiploic vascular arc were preserved. We ligated and divided the right gastric artery whenever this extensive mobilization was inadequate. The diaphragmatic crura and vagal trunks were cut after division of the left gastric artery and vein, with mobilization of the stomach so that the pylorus reaches the diaphragmatic level. ${ }^{10-13}$ The esophagus was mobilized from the mediastinum by blunt finger dissection. Pyloromyotomy was a routine step in all operations. After completing the mediastinal dissection, we mobilized the upper thoracic esophagus under the guidance of cervical esophagus Figure(2).

In all cases, we performed several applications of GIA stapler along the lesser curvature to provide a gastric tube with greatest possible mobility Figure(3A). The tube was pulled up to the base of the mouth through the posterior mediastinal way, accomplishing the anastomosis between the tube and the base of the mouth with an interrupted full thickness layer of $3 / 0$ vicryl stitch Figure $(3 \mathbf{B} \& \mathbf{C})$.

We closed the wounds over two vacuum drains in the neck and one vacuum drain in the abdominal cavity after constructing a feeding jejunostomy. Contrast radiography was obtained on the 9th postoperative day Figure(4).

All the patients were transferred intubated to the intensive care unit.

\section{Results:}

This study included 12 patients ( 8 males, 4 females) with mean age of 55 (range 49-65) years. The diagnosis in all of them was hypopharyngeal (postcricoid) carcinoma. Two patients with neck nodes larger than $6 \mathrm{~cm}$ received preoperative radiotherapy.

According to the extent of the tumors on either side of the neck, the otorhinolaryngology surgeons carried out radical or modified radical neck dissections together with the pharyngolaryngectomy. The operation time in total was $460+/-140$ minutes, and tended to become shorter as we gained familiarity with 

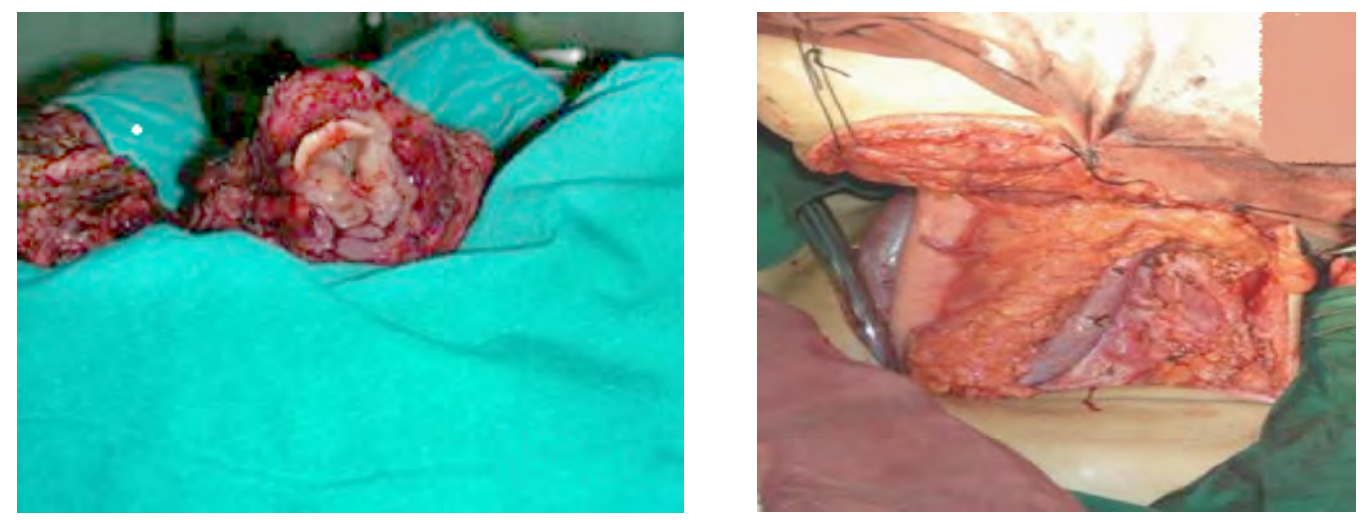

Figure (1): A-Pharyngolaryngectomy with B-Modified radical neck dissection.

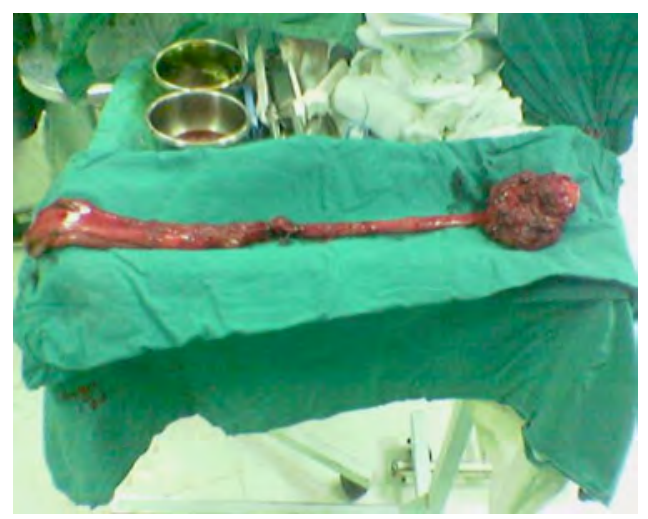

Figure (2): Pharyngolaryngectomy plus thoracic esophagectomy.
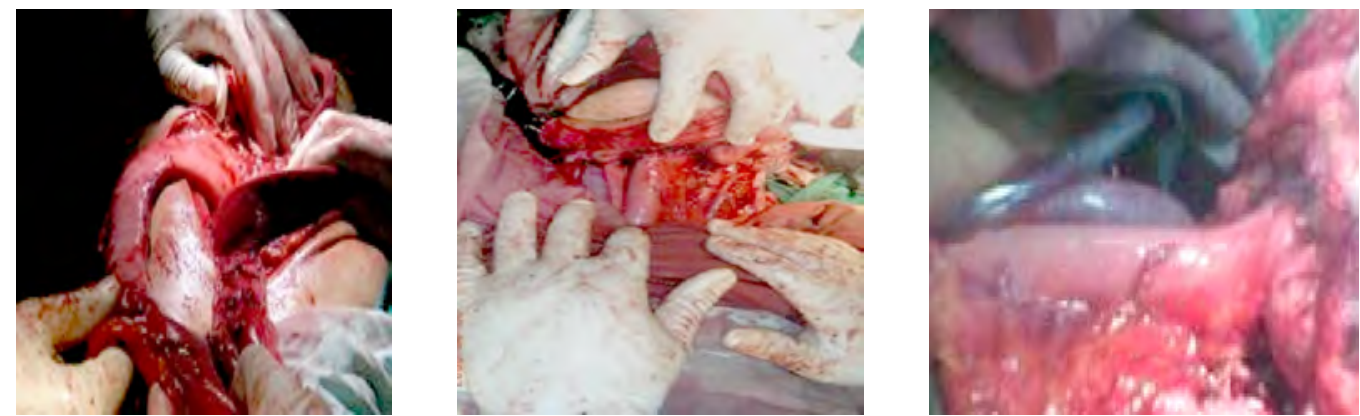

Figure (3): A- Construction of gastric tube. $B \& C$-Single layer anastomosis between the tube and base of mouth.

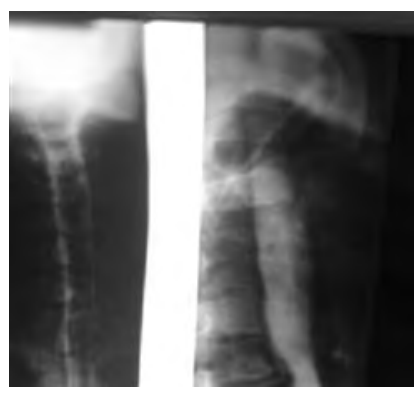

Figure (4): Postoperative dye study with normal passage of contrast. 
the technique. Major anastomotic leak occurred in two patients; one of them received perioperative radiotherapy, whereas the resection margin was infiltrated in the other as disclosed by the postoperative histopathology. Both of them underwent reanastomosis.

Radiological study detected minor leak in two patients. Neck hematoma occurred in one patient who needed neck exploration 12 hours postoperatively with diathermy control of a small bleeder.

Total mortality was $5 / 12$. Two patients with major leak died within 2 weeks of the re-do. The causes of death were mainly related to respiratory failure (autopsy not done). One patient who needed neck exploration for a hematoma died following the sudden development of pneumothorax and consequent myocardial infarction while ventilated on the fifth postoperative day and two patients died from severe electrolyte disturbance and multiorgan failure on the seventh postoperative day. Cardiac rhythm abnormalities in the form of insignificant ectopic beats were observed in five patients, especially early postoperatively. This required no intervention and disappeared spontaneously. Pneumonia developed in three patients and resolved with appropriate conservative measures.

We noted no swallowing problems in five of the surviving seven patients. The remaining two patients with minor leak complained of mild to moderate dysphagia $35 \& 45$ days postoperatively, underwent endoscopic dilatation utilizing Savary dilators after 3 months of the operation, and repeated after one month. They did not show up again, arbitrary indicating the success of the dilatation.

Apart from these two patients presented with dysphagia, we did not perform routine endoscopic or radiological surveillance for the remaining patients. The mean follow-up period was 7 months (range 6-24 months). All the seven surviving patients complained of regurgitation of various severities following the start of oral intake.

\section{Discussion:}

Hypopharyngeal carcinoma is prone to occur in patients with history of heavy alcohol ingestion and heavy smoking. ${ }^{14}$ Common presentations are persistent dysphagia, persistent sore throat, or a foreign body sensation in the throat. Pain referred to the ear by Arnolds nerve, a division of 10th nerve is a later symptom. Twenty percent of patients have an asymptomatic mass in the neck, usually a jugulodigastric or midjugular lymph nodes. Associated symptoms include progressive weight loss, hemoptysis, and hoarseness of voice. ${ }^{11}$

Indirect laryngoscopic examination is the first tool used to examine the patients. An ipsilateral fixed cord suggests a more advanced hypopharyngeal tumor. There may be asymmetry of the laryngeal structure and edema in the arytenoid region. Flexible fiberoptic laryngoscopy may be necessary when mirror examination is inadequate

When a malignancy of the hypopharynx is suspected, CT and MRI are preferably done before direct endoscopic examination with biopsy to avoid radiological misinterpretations. CT may draw attention to additional areas that need assessment and require biopsy at time of endoscope. On the other hand, MRI gives very clear information about soft tissue and neurovascular invasion. ${ }^{11}$

Endoscopic assessment of the hypopharynx is done under general anesthesia, not only to take biopsies but also to determine the mobility of the tumor over the prevertebral fascia. Endoscopy also rules out the existence of a second or concurrent malignancy and ensures that no skip lesions exist. ${ }^{11}$

Based on the preoperative staging work up mentioned above, the followings are indications for unresectability: Presence of fixed cervical metastases, encasement or involvement of the carotid artery, fixation of the posterior pharyngeal wall (prevertebral fascia invasion), extension into the thoracic esophagus, and evidence of distant metastatic disease. ${ }^{11}$

All the patients selected for surgery in our study fulfilled both the radiological and endoscopic criteria for resectability.

In the early 1960s, surgery was the primary modality for managing malignancies of the hypopharynx once the patient was fit for surgery. Preoperative radiotherapy combined with surgical resection became the most popular 
management during the late 1960s and early 1970s. ${ }^{15}$

Hypopharyngeal (postcricoid) carcinomas show frequent metastases to the surrounding tissues and regional lymph nodes, resulting in $75 \%$ early recurrences and 5-year survival rate of $25 \% .13-15 \mathrm{In}$ addition to the recurrence and low survival rates after the resection of these tumors, the difficulty in restoring continuity between the base of the mouth and the gastrointestinal tract increases morbidity and may be an additional cause of mortality.

The use of skin flaps and myocutaneous flaps for the reconstruction of the resultant defect are generally not recommended because they are multi-stage operations and have high incidences of fistula and stricture. ${ }^{2}$ Moreover, for starting oral nutrition of the patients, 2-3 months are required postoperatively, causing additional morbidity. ${ }^{6}$ If there is no chance for proper TPN or if TPN complications develop, gastrostomy or feeding jejunostomy is needed for this group of patients. The morbidities of these procedures are thus added to the picture. Skin flaps and myocutaneous flaps also cause serious cosmetic defects. ${ }^{5}$

Other reconstructive methods include colon interposition and jejunal free flaps. Colon interposition requires complete dissection and mobilization of a segment of the colon, and by definition, the relevant colonic mesentery also needs to be transposed. ${ }^{16,17}$ This may cause circulation disorders resulting from tension and/or pressure in the mediastinal, subcutaneous, or retrosternal tunnel. There are other objections such as the low thickness of the colonic wall or anastomotic ulcer, and the inevitable addition of colo-colic anastomosis in the abdomen. ${ }^{18}$

Jejunal flap is suggested as an ideal method for esophageal replacement, especially in the cases with a former stomach operation. ${ }^{19}$ However, jejunal free flap requires, in addition to three anastomoses, a refined microsurgery technique for the application of vascular anastomosis that may be at risk especially in patients who received preoperative radiotherapy. ${ }^{8}$ Furthermore, the rate of stenosis in jejunal flaps is reported to be higher than in the gastric pull-up method. ${ }^{20}$

Gastric pull-up method has been identified as the easiest and lowest risk method as it requires a single anastomosis at the base of the mouth, which is readily accessible. ${ }^{10,12,13}$ Extensive mobilization of the stomach is needed, so that the pylorus reaches the level of the hiatus. Due to the rich vascular supply of the stomach, this mobilization has never caused vascular problems in our cases. Whenever this mobilization proves to be inadequate, the right gastric artery can be divided, and the vascular supply of the gastric tube then relies wholly on the right gastroepiploic artery and vein. Pyloromyotomy was the rule rather than the exception among our patients in an attempt to evade the possible delayed emptying of the gastric tube with the consequent deglutatory difficulties. We conducted the dissection through the mediastinum in a completely blind manner, as in blunt esophagectomy. Following total replacement of the esophagus with the gastric tube into the neck, pharyngo-gastrostomy was accomplished.

The major reported drawbacks of the gastric pull-up method are postoperative respiratory problems, cardiac arrhythmia, loss of weight on the long term, and serious regurgitation. ${ }^{13}, 20$ Four mortalities in our patients related to the respiratory system happened in the early postoperative period in the ICU. We observed cardiac rhythm abnormalities in the form of insignificant ectopic beats early postoperatively in five patients. This required no intervention and disappeared spontaneously. The pressure of the gastric tissue placed in the posterior mediastinum is believed to play a role in this dysrrhythmia. ${ }^{12}$ Three patients developed pneumonia, aggressively treated conservatively.

The most important late complication was regurgitation that occurred in all our surviving patients when lying on their backs. We warned the patients about the possibility or even the inevitability of its occurrence, and encouraged them to take regular meals and not to lie on their backs for 2-3 hours after oral intake. Persistent symptoms were difficult to treat and we reassured the patients about the benign nature of this annoying complaint.

Long-term postoperative weight loss due to absence of the physiologic role of stomach in normal nutrition was difficult to gauge in 
this work with a mean follow up of only seven months. In addition, the patients were already malnourished and striving for an access to swallow.

We anticipated the two mild strictures in this series as they occurred in the patients who developed minor leak. The strictures were dilated twice with Savary dilators and we assume it was sufficient, as the patients did not return. Frank leaks occurred in two patients one who received preoperative radiotherapy and another patient with positive resection margin in the orad side of the specimen. We did not know the result of the redo in these two patients as they succumbed to their operations.

According to our preliminary results, gastric pull-up reconstruction appears to be an efficient and relatively safe method of reconstruction after radical resections for postcricoid tumors.

\section{Conclusion:}

Gastric pull up is a major operation in frail malnourished patients. Despite the high mortality attended with this operation in our work, it seems to be the best among other alternatives at least because of the lower possibility of stricture and the need for a single anastomosis.

\section{References:}

1- Carpenter R, DeSanto W and Devine K: Reconstruction after total laryngopharyngectomy. Arch Otolaryngol 2003; 105: 417-422.

2- Bakamjian V, Ciano M: The reconstructive role in cancer surgery of the head and neck. Ann Chir Plast 2001; 27: 133-136.

3- Gilas T, Sako K, Calamel M: Major Head and neck reconstruction using the deltopectoral flap. A 20-year experience. Am J Surg 2005; 152: 430-434.

4- Ariyan S: The pectoralis major myocutaneous flap. A versatile flap for reconstruction in the head and neck. Plast Reconstr Surg 2006; 63: 73-81.

5- Schuller D: Reconstructive options for pharyngeal and/or cervical esophageal defects. Otolaryngol 2004; 111: 193-197.

6- Rees R, Ivey G, Lynch B: Pectoralis major musculocutaneous flaps: Long-term follow- up of hypopharyngeal reconstruction. Plast Reconstr Surg 2004; 77: 586-591.

7- Seidenberg B: Immediate reconstruction of the cervical esophagus by a revascularized isolated jejunal segment. Ann Surg 2000; 194: 162-171.

8- Fisher S, Cole T, Seigler H: Pharyngoesophageal reconstruction using free jejunal interposition grafts. Arch Otolaryngol 2005; 111: 747-752.

9- Shangold L, Urken M, Lawson W: Jejunal transplantation for pharyngoesophageal reconstruction. Otolaryngol Clin North Am 2005; 24: 1321-1342.

10 -Le Quesne L, Ranger D: Pharyngolaryngectomy with immediate pharyngogastric anastomosis. Br J Surg 2002; 53: 105-109.

11-Bova R, Goh R, Poulson M, Coman WB: Total pharyngolaryngectomy for squamous cell carcinoma of the hypopharynx: A review. Laryngoscope 2005; 115(5): 8649.

12-Spiro R, Bains M, Strong W: Gastric transposition for head and neck cancer: A critical update. Am J Surg 2002; 162: 348352.

13-Cahow C, Sasaki T: Gastric pull-up reconstruction for pharyngo-laryngoesophagectomy. Arch Surg 2005; 129: 425429.

14-Guenel P, Chastang JF, Luce D, Bottrill ID, Howard DJ: A study of the interaction of alcohol drinking and tobacco smoking among French cases of laryngeal cancer. J Epidemiol Community Health 1988; 42(4): 350-4.

15-Axon PR, Woolford TJ, Hargreaves SP, Beauvillain C, Mahe M, Bourdin S : A comparison of surgery and radiotherapy in the management of post- cricoid carcinoma. Clin Otolaryngol 1997; 22(4): 370-4.

16-Davis PA, Law S, Wong J: Colonic interposition after esophagectomy for cancer. Arch Surg 2003; 138(3): 303-8.

17-Yildirim S, Köksal H, Celayir F, Erdem L, Oner M, Baykan A: Colonic interposition vs. gastric pull-up after total esophagectomy. J Gastrointest Surg 2004; 8(6): 675-8.

18-Cense HA, Visser MR, van Sandick JW, 
de Boer AG, Lamme B, Obertop H, et al: Quality of life after colon interposition by necessity for esophageal cancer replacement. J Surg Oncol 2004; 88(1): 32-38.

19-Kamei S, Takeichi Y, Baba S: Reconstruction using a free jejunal graft for surgery of the hypopharynx and the cervical esophagus in patients with a history of previous upper gastro-intestinal surgery. Acta Otolaryngol 2004; 525(Suppl): 3539.
20-Kato H, Watanabe H, Harii K: Primary esophageal reconstruction after resection of the cancer in the hypopharynx or cervical esophagus: Comparison of free forearm skin tube flap, free jejunal transplantation and pull-through esophagectomy. Jpn J Clin Oncol 2003; 17: 255-261. 